\title{
Singular dynamics under a weak potential on a sphere
}

\author{
Roberto Castelli, Francesco Paparella and Alessandro Portaluri
}

\begin{abstract}
We give a detailed analytical description of the global dynamics of a point mass moving on a sphere under the action of a logarithmic potential. We perform a McGehee-type blow-up in order to cope with the singularity of the potential when the point mass goes through the singularity. In addition we investigate the rest-points of the flow, the invariant (stable and unstable) manifolds and we give a complete dynamical description of the motion.
\end{abstract}

Mathematics Subject Classification. Primary 70F10; Secondary 37C80.

Keywords. Singular dynamics, McGehee coordinates, Regularization of collisions, Heteroclinics.

\section{Contents}

1. Introduction

2. Preliminaries

3. The stereographic projection of the sphere

4. Position of the problem

5. Energy hypersurfaces, regularization and flow

6. Global flow and dynamics on the sphere

Acknowledgements

$\begin{array}{lll}\text { Appendix A. Useful limits } & 869\end{array}$

References

\section{Introduction}

Topologically, two dimensional Riemann surfaces with constant (Gaussian) curvature $K$ are classified into three categories: Euclidean spheres, $\mathbb{S}^{2}(K>0)$; Euclidean planes, $E^{2}(K=0)$; and hyperbolic planes $H^{2}(K<0)$. Among

Work partially supported by "Progetto 5 per mille per la ricerca" (Bando 2011). "Collisioni fra vortici puntiformi e fra filamenti di vorticità: singolarità, trasporto e caos." 
them, $\mathbb{S}^{2}$ and $E^{2}$ are more familiar and come out very often in practice. For example, the mechanics of thin fluid layers on $\mathbb{S}^{2}$ provides a global model of a planetary atmosphere, and that on $E^{2}$ is its local approximation.

In this paper we consider the one-center logarithmic potential problem on a sphere, that is the dynamics of a point particle moving on a sphere under the action of a logarithmic central potential. More formally, we choose a point $Q \in S^{2}$ as the center of the potential, and we assume that at any point $x \in \mathbb{S}^{2}, x \neq Q$ the particle is subjected to one of the two following forces:

1. $f_{\text {geo }}(P)=k \nabla\left(-\log \left(d_{\boldsymbol{S}}(P, Q)\right)\right.$, where $d_{\boldsymbol{S}}(P, Q)$ is the geodesic distance between the points $P$ and $Q$.

2. $f_{\text {euc }}(P)=k \nabla(-\log (d(P, Q))$ where $d(P, Q)$ is the euclidean distance between the points $P$ and $Q$.

The sign of the constant $k$ determines whether the center $Q$ is attracting or repelling.

Two are the main reasons for the choice of these potentials. First, they arise in different physical scenarios: such as in models of astrodynamics [18, 19]; in the dynamics of a charged particle in a cylindrically symmetric electric field [13] and in the mathematical theory of vortex filaments of an ideal fluid $[14,17]$. The second reason relies on the fact that the logarithmic potential $V(x)=-\log (|x|)$ may be considered a sort of limit case for $\alpha \rightarrow 0$ of the homogeneous potentials $V_{\alpha}=|x|^{-\alpha}$. While the latter have been extensively studied by different authors, the former has not been so deeply investigated. In particular one could be interested to know if (and how) some features regarding, for instance, the regularization of collisions, the minimality properties of the solutions and their stability, may be extended from the homogeneous to the logarithmic potential case. Results in this direction have been achieved for instance in $[2,3,19]$.

In addition, we consider a sphere, rather than the classical two or threedimensional Euclidean space, as the configuration space. Our goal is to understand which aspects of the dynamics are affected if the geometry of the underlying space changes, or, equally well, what survives of the planar dynamics if one considers a curved manifold. The study of the motion of particles on curved space is a classical problem. Here we only quote recent papers [5-9] where, among others interesting features, the relative equilibria and homographic solutions of the classical $n$-body potentials on curved manifolds have been investigated. For further references on the subject the reader is referred to the references in those papers.

From a dynamical point of view the most interesting role, and the hardest obstacle for a full understanding of the motion, is played by the presence of the singularity in the potential function. Indeed, as it often happens in celestial mechanics, the singularities are the source of a complicated dynamics. From the point of view of the analysis, the singularities represent a severe technical hurdle to overcome and different techniques have been proposed to regularize the vector field, mainly for the homogeneous potential case $[3,4,10,12,15,16]$.

This paper is inspired by the recent work [18] that studies the planar motion of a point mass subject to a logarithmic potential in an astrodynamic 


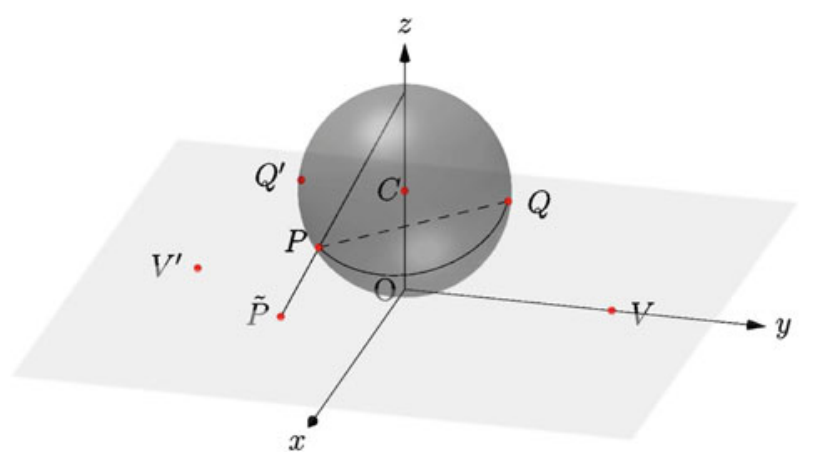

FiguRE 1. Mutual positions of the sphere (centered at the point $C$ ) and of the stereographic plane. The point $Q$ is the center of the logarithmic potential; $Q^{\prime}$ is its antipodal point; $V$ and $V^{\prime}$ are the stereographic projections of, respectively $Q$ and $Q^{\prime}$. The point subject to the force is $P$, whose stereographic projection is $\tilde{P}$. The length of the dashed segment joining $P$ and $Q$ is the Euclidean distance $d(P, Q)$; the length of the solid arc is the geodetic distance $d_{\boldsymbol{S}}(P, Q)$

context. To overcome the singularity of the vector field we adapt to our problem the celebrated McGehee transformation, a regularizing change of variables currently popular in the field of Celestial Mechanics and first introduced in 1974 by McGehee [16] to solve the collisions in the collinear three-body problem.

The McGehee transformations consist of a polar type change of coordinates in the configuration space, together with a suitable rescaling of the momentum. In this way the total collision is blown-up into an invariant manifold called total collision manifold over which the flow extends smoothly. Furthermore, each hypersurface of constant energy has this manifold as a boundary. By rescaling time in a suitable way, it is possible to study qualitative properties of the solutions close to total collision, obtaining a precise characterization of the singular solutions.

The McGehee transformations are usually applied to the case of homogeneous potentials (see, for instance, [11]) but, as shown in [18] and as it will be manifest throughout this paper, with slight modifications they give interesting results even in the presence of a logarithmic potential. In fact, although the lack of homogeneity of the logarithmic nonlinearity breaks down some nice and useful properties of the transformation, it is still possible to regularize the vector field and to carry out a detailed analytical description of the rest points, of the invariant manifolds, and of the heteroclinics on the collision manifold. The last are indeed the features that allow to extend the singular solutions beyond the singularity so that the extended flow results continuous with respect to initial data.

Let us now formalize the problem, define the notations and summarize the results. 
Denote with $S$ the two-dimensional sphere of radius $R$ and center $C=$ $(0,0, R)$, namely

$$
\boldsymbol{S}:=\left\{(x, y, z) \in \mathbb{R}^{3}: x^{2}+y^{2}+(z-R)^{2}=R^{2}\right\}
$$

where $(x, y, z)$ are the Cartesian coordinates in $\mathbb{R}^{3}$ (Fig. 1 ).

We use slightly non-standard angular coordinates $\phi$ and $\theta$ for the spherical surface: to any point $P \in \boldsymbol{S}$ are associated the coordinates

- $\phi \in[0,2 \pi)$ the usual polar angle of the projection of $P$ onto the plane $z=0$;

- $\theta \in[0, \pi]$ the angle $\widehat{O C P}$, where $O$ is the origin of the Cartesian reference frame.

For ease of language we shall call $Q$ as the vortex point, the half-sphere centered around the vortex point as the vortex half-sphere, and the complementary half-sphere antivortex half-sphere; let us call vortex-parallel any circle on the sphere equidistant from $Q$ and vortex-meridian any great circle passing through $Q$. Moreover, we name $Q^{\prime}$ the antipodal point, that is the point on the sphere opposite to the vortex point.

In order to illustrate some basic results with the maximum clarity, for the moment we won't place the vortex point as in Fig. 1, but at the north pole, i.e. $Q=(0,0,2 R)$, so that the curves $\{\theta=$ const $\}$ and $\{\phi=$ const $\}$ respectively correspond to the vortex parallels and to the vortex meridians. Of course, the dynamics is not affected by this choice, which is just a temporary convenience. In this setting, the geodesic distance is simply $d_{\boldsymbol{S}}(P, Q)=R(\pi-\theta)$, yielding the Hamiltonian

$$
\mathcal{H}_{\text {geo }}\left(\theta, \phi, p_{\theta}, p_{\phi}\right)=\frac{1}{2 R^{2}}\left(\frac{1}{\sin ^{2} \theta} p_{\phi}^{2}+p_{\theta}^{2}\right)+\frac{\Gamma}{4 \pi} \log (R(\pi-\theta)) .
$$

In the attractive case, $\Gamma>0$, let us define $h_{1}=\frac{\Gamma}{4 \pi} \log \left(\frac{\pi}{2} R\right), h_{2}=$ $\frac{\Gamma}{4 \pi} \log (\pi R)$. The analysis of the energy shells to be performed in Lemma 5.1 will allow us to conclude that

- if $h \geq h_{2}$ then the motion is allowed everywhere on the sphere;

- if $h<h_{2}$ the motion is allowed only in a sphere cap containing the vortex point whose boundary is a vortex-parallel that lies on the vortex half-sphere for $h<h_{1}$ or in the antivortex half-sphere if $h_{2}<h<h_{1}$.

Besides the energy $h$, it is easy to show that there exists a second conserved quantity, namely the spherical angular-momentum, $l$. In Lemmas 6.2 and 6.3 we show that

- A necessary condition in order to have a collision solution or to reach the antipodal point is $l=0$.

- Any vortex-parallel on the vortex half-sphere is the support of a periodic orbit.

- The vortex-meridians are flow-invariant.

Figure 2 depicts some of the solutions lying on vortex parallels and on vortex-meridians 


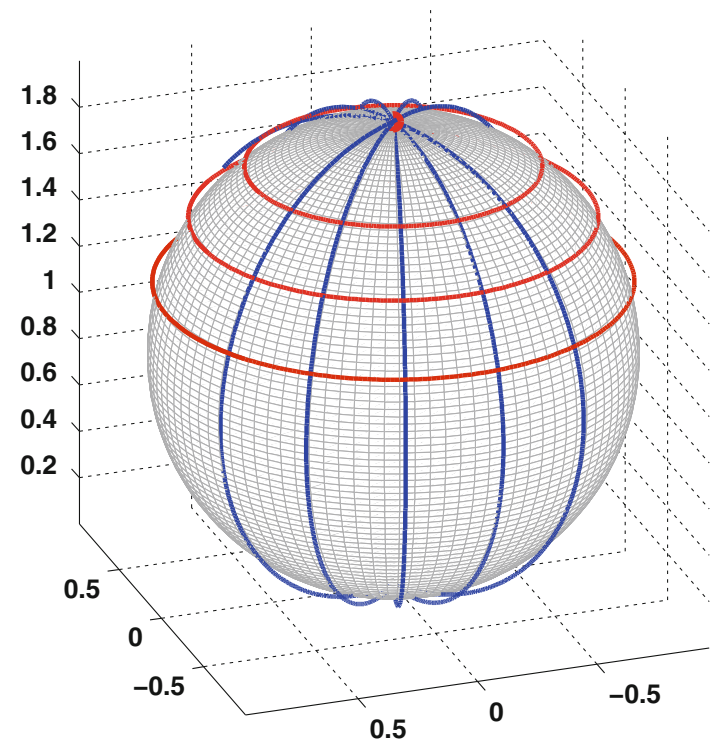

Figure 2. Periodic orbits lying on the vortex-parallels and collision orbits lying on the vortex-meridians

As a consequence, collision with the vortex point is possible if and only if the particle moves along a meridian. The main question that we address in the paper is whether it is possible to extend such a trajectory beyond the singularity so that the extended flow admits a regularity of some sort. The study of the heteroclinic connections between invariant manifolds in McGehee coordinates provides a way to formulate a positive answer. Let us define the notion of collision-transmission solution:

Definition 1.1. Let $\left(\phi_{V}, \theta_{V}\right)$ be the coordinates of the vortex point and let $\gamma(t)=(\phi, \theta)(t):\left[0, T_{s}\right) \rightarrow \boldsymbol{S}$ be a collision solution ending in the singularity at time $T_{s}$. We define as collision-transmission trajectory the path $\bar{\gamma}:\left[0,2 T_{s}\right] \rightarrow \boldsymbol{S}$ given by

$$
\bar{\gamma}(t):=\left\{\begin{array}{ll}
\gamma(t), & t \in\left[0, T_{s}\right) \\
\left(\phi_{V}, \theta_{V}\right) & t=T_{s} \\
\left(2 \phi_{V}-\phi\left(2 T_{s}-t\right), 2 \theta_{V}-\theta\left(2 T_{s}-t\right)\right) & t \in\left(T_{s}, 2 T_{s}\right]
\end{array} .\right.
$$

Based on the previous definition, we prove that

Theorem 1.1. The flow obtained by extending the collision solutions with the collision-transmission trajectories is continuous with respect to initial data anywhere out of the vortex and of the antipodal points.

The results for the problem with the Euclidean distance are almost completely analogous, with a noteworthy increase of regularity at the antipodal point. Throughout the paper we will point out any other difference that will occur. 
Finally, let us observe that the problem we are dealing with is integrable. Regular trajectories might be studied just with the Hamiltonian (1). Difficulties arise for the collision trajectories, or those very close to them. In this case an approach based on McGehee-like transformations appears to be the only possible way to attack the problem. This, in turn, requires a few words about the location of the vortex point, and on the use of the stereographic projection. It appears natural to place the vortex in correspondence to one of the poles of the coordinate system on the sphere but this choice will make the singularity of the potential and the singularity of the kinetic energy matrix to interfere with each other in such a way that the McGehee regularization could not be performed. On the other hand, if we place the source of the potential away from the poles of the coordinate system, then we (obviously) loose a cyclical coordinate, but this is a small price to pay for the ability to perform the regularization in a relatively straightforward way. In addition, it turns out that transformations of the McGehee type may be devised without too many difficulties for equations which are written in Cartesian coordinates on a plane. Therefore, rather than attempting to work directly onto the sphere, we felt it would be more easy (and more clear) first to project the dynamics on a stereographic plane, and then to remove the singularities of the resulting equations. We choose the stereographic projection just because we appreciate the possibility of using a single map for the whole sphere except a point. Other choices would not change significantly the difficulty of the problem. We are confident that this general scheme may be effective for many other singular problems on more general manifolds.

The paper is organized as follows: first we recall some basic notions about the Hamiltonian formulation of the co-geodesics flow on a general Riemannian manifold, then in Sect. 3 we restrict to the case of the sphere and we formulate the equivalent co-geodesics flow through the stereographic projection. In Sect. 4 we introduce the singular logarithmic potential and we write the equations of motion. Section 5 deals with the in-deep study of the dynamical system: we regularize the singularity of the potential with the modified McGehee technique, and we provide an analysis of the flow on the collision and on the zero velocity manifolds. Section 6 concerns the global dynamics and we rephrase the results in terms of the original motion on the sphere with untransformed coordinates.

\section{Preliminaries}

Let $(M, g)$ be a $n$-dimensional Riemannian manifold. We denote by $D$ the associated Levi-Civita connection and by $\frac{\mathrm{D}}{\mathrm{dt}}$ the covariant derivative of a vector field along a smooth curve $\gamma$. Let $I$ be an interval of the real line and $V$ be a smooth function defined on $I \times M$.

Definition 2.1. A perturbed geodesic abbreviated as p-geodesic is a smooth curve $\gamma: I \rightarrow M$ which satisfies the differential equation 


$$
\frac{\mathrm{D}}{\mathrm{dt}} \gamma^{\prime}(t)+\nabla V(t, \gamma(t))=0
$$

where $\nabla V$ denotes the gradient of $V(t,-)$ with respect to the metric $g$.

Remark 2.2. From a dynamical viewpoint, the pair $(g, V)$ define a mechanical system on the manifold $M$, with kinetic energy $\frac{1}{2} g(v, v)$ and time dependent potential energy $V$. Solutions of the differential equation (2) correspond to trajectories of particles moving on the Riemannian manifold in the presence of the potential $V$. If the potential vanishes we get trajectories of free particles and hence geodesics on $M$. This motivates the suggestive name, "perturbed geodesics" in the case $\nabla V \neq 0$. Moreover, if the potential $V$ is time independent, modulo reparametrization, perturbed geodesics become geodesics of the Jacobi metric associated to $(g, V)$ : indeed the total energy

$$
e=\frac{1}{2} g(\gamma(t))\left(\gamma^{\prime}(t), \gamma^{\prime}(t)\right)+V(\gamma(t))
$$

is constant along any trajectory $\gamma$. Therefore, whenever $V$ is bounded from above, the solutions of (2) with energy $e>\sup _{m \in M} V(m)$ are just reparametrized geodesics for the metric $[e-V] g$ on $M$ with total energy equal to one [1].

Denoting by $\left(q^{1}, \ldots, q^{n}\right)$ a local system of coordinates on $M$, equation (2) reduces to

$$
\ddot{q}^{i}+\Gamma_{j k}^{i} \dot{q}^{j} \dot{q}^{k}=-g^{i j} \frac{\partial V}{\partial q^{j}},
$$

where, as usual, $g^{i j}=(g)_{i j}^{-1}$, and $\Gamma_{j k}^{i}$ are the Christoffel symbols.

\section{Geodesic flow as Hamiltonian flow}

The geodesic flow turns out to be a Hamiltonian flow of a special Hamiltonian vector field defined on the cotangent bundle of the manifold. The Hamiltonian depends on the metric on the manifold and it is a quadratic form consisting entirely of the kinetic term. The geodesic equation corresponds to a secondorder nonlinear ordinary differential system. Therefore, by suitably defining the momenta, it can be re-written as first-order Hamiltonian system.

More explicitly, let us consider a local trivialization chart of the cotangent bundle $T^{*} M$

$$
\left.T^{*} M\right|_{U} \cong U \times \mathbb{R}^{n}
$$

where $U$ is an open subset of the manifold $M$, and the tangent space is of rank $n$. Let us denote by $\left(q_{1}, q_{2}, \ldots, q_{n}, p_{1}, p_{2}, \ldots, p_{n}\right)$ the local coordinates on $T^{*} M$ and introduce the Hamiltonian

$$
H: T^{*} M \rightarrow \mathbb{R}: H(\boldsymbol{q}, \boldsymbol{p})=\frac{1}{2} g^{i j}(\boldsymbol{q}) p_{i} p_{j} .
$$

The Hamilton-Jacobi equations of the geodesic equation with respect to the metric $\boldsymbol{g}$ can be written as 


$$
\left\{\begin{array}{l}
\dot{q}^{i}=\frac{\partial H}{\partial p_{i}}=g^{i j}(\boldsymbol{q}) p_{j} \\
\dot{p}_{i}=-\frac{\partial H}{\partial q_{i}}=-\frac{1}{2} \frac{\partial g^{j k}}{\partial q^{i}} p_{j} p_{k} .
\end{array}\right.
$$

The second order geodesic equations are easily obtained by substitution of one into the other. The flow determined by these equations is called the co-geodesic flow, while the flow induced by the first equation on the tangent bundle is called geodesic flow. Thus, the geodesic lines are the projections of integral curves of the geodesic flow onto the manifold $M$.

Being the Hamiltonian $H$ time-independent, it is readily seen that it is constant along the geodesics. Thus, the co-geodesic flow splits the cotangent bundle into level sets of constant energy

$$
M_{E}=\left\{(\boldsymbol{q}, \boldsymbol{p}) \in T^{*} M: \quad H(\boldsymbol{q}, \boldsymbol{p})=E\right\},
$$

for each energy $E \geq 0$, so that

$$
T^{*} M=\bigcup_{E \geq 0} M_{E}
$$

Now let $\boldsymbol{g}, \boldsymbol{h}$ be two Riemannian metrics on $M$ in the same conformal class; namely there exists a positive and smooth function $\lambda=\lambda(\boldsymbol{q})$ of the coordinates such that

$$
\boldsymbol{g}^{i j}=\lambda \boldsymbol{h}^{i j}
$$

or equivalently $\boldsymbol{g}=\lambda^{-1} \boldsymbol{h}$. From the definition (3) it follows that a scaled co-geodesic Hamiltonian function corresponds to a conformal change of the metric. In fact if $H_{\boldsymbol{g}}$ and $H_{\boldsymbol{h}}$ denote the Hamiltonian co-geodesic functions and if $\boldsymbol{g}, \boldsymbol{h}$ are in the same conformal class then it immediately follows by (3) that

$$
H_{\boldsymbol{g}}(\boldsymbol{q}, \boldsymbol{p})=\lambda H_{\boldsymbol{h}}(\boldsymbol{q}, \boldsymbol{p}) .
$$

As a consequence, Hamilton's equations with respect to this two Hamiltonian functions are related as follows

$$
\left\{\begin{array}{l}
\dot{\boldsymbol{q}}=\partial_{\boldsymbol{p}} H_{\boldsymbol{g}}=\lambda \partial_{\boldsymbol{p}} H_{\boldsymbol{h}}+H_{\boldsymbol{h}} \partial_{\boldsymbol{p}} \lambda=\lambda \partial_{\boldsymbol{p}} H_{\boldsymbol{h}} \\
\dot{\boldsymbol{p}}=-\partial_{\boldsymbol{q}} H_{\boldsymbol{g}}=-\lambda \partial_{\boldsymbol{q}} H_{\boldsymbol{h}}-H_{\boldsymbol{h}} \partial_{\boldsymbol{q}} \lambda
\end{array}\right.
$$

where the last equality in the first equation comes from the fact that the function $\lambda$ only depends on $\boldsymbol{q}$. In the following we consider a perturbed-geodesic flow on a sphere, thus it is worth to write down explicitly the free geodesic flow when the manifold $M$ is a surface of revolution in $\mathbb{R}^{3}$.

Let us denote with $(x, y)$ the Cartesian coordinates of $\mathbb{R}^{2}$ and let us consider the function $\varphi: U \subset \mathbb{R}^{2} \rightarrow \mathbb{R}^{3}$ given by $\varphi(x, y)=(f(y) \cos x, f(y) \sin x$, $g(y))$

$$
U=\left\{(x, y) \in \mathbb{R}^{2}: 0 \leq x<2 \pi, y_{0}<y<y_{1}\right\},
$$

where $f$ and $g$ are differentiable functions, with $f^{\prime}(y)^{2}+g^{\prime}(y)^{2} \neq 0$ and $f(y) \neq$ 0 . Thus $\varphi(x, y)$ is an immersion and the image $\varphi(U)$ is the surface generated 
by the rotation of the curve $(0, f(y), g(y))$ around the $z$ axis. ${ }^{1}$ The induced Riemannian metric $g=\left(g_{i j}\right)$ in the $(x, y)$ coordinates is given by

$$
g_{11}=f^{2} \quad g_{12}=0 \quad g_{22}=\left(f^{\prime}\right)^{2}+\left(g^{\prime}\right)^{2} .
$$

From (3), the Hamiltonian function associated to the geodesic flow is given by

$$
H\left(x, y, p_{x}, p_{y}\right)=\frac{1}{2}\left(\frac{1}{f^{2}} p_{x}^{2}+\frac{1}{f^{\prime 2}+g^{\prime 2}} p_{y}^{2}\right)
$$

and the co-geodesics flow reads as

$$
\left\{\begin{array}{l}
\dot{x}=\frac{1}{f^{2}} p_{x} \\
\dot{y}=\frac{1}{f^{\prime 2}+g^{\prime 2}} p_{y} \\
\dot{p}_{x}=0 \\
\dot{p}_{y}=\left[\frac{f f^{\prime}}{f^{4}} p_{x}^{2}+\frac{f^{\prime} f^{\prime \prime}+g^{\prime} g^{\prime \prime}}{\left(f^{\prime 2}+g^{\prime 2}\right)^{2}}\right]
\end{array}\right.
$$

or, equivalently, as

$$
\left\{\begin{array}{l}
\ddot{x}+\frac{2 f f^{\prime}}{f^{2}} \dot{x} \dot{y}=0 \\
\ddot{y}-\frac{f f^{\prime}}{f^{\prime 2}+g^{\prime 2}}(\dot{x})^{2}+\frac{f^{\prime} f^{\prime \prime}+g^{\prime} g^{\prime \prime}}{f^{\prime 2}+g^{\prime 2}}(\dot{y})^{2}=0 .
\end{array}\right.
$$

\section{The stereographic projection of the sphere}

\section{Stereographic projection}

The contents of this subsection are completely standard and the exposition is mainly devoted to fix some notations. We have already introduced the sphere $\boldsymbol{S}$

$$
\boldsymbol{S}:=\left\{(x, y, z) \in \mathbb{R}^{3}: x^{2}+y^{2}+(z-R)^{2}=R^{2}\right\}
$$

and we call north pole and south pole the point $N:=(0, \ldots, 0,2 R) \in \boldsymbol{S}$ and its antipodal $S:=(0, \ldots, 0,0) \in \boldsymbol{S}$, respectively. Note that the sphere is tangent at the origin $O$ to the plane $\{\boldsymbol{z}=0\}$, that we identify with $\mathbb{R}^{2}$ (Fig. 1). Denote by $\pi_{S}$ the stereographic projection

$$
\begin{aligned}
\pi_{S}: S \backslash\{N\} & \longrightarrow \mathbb{R}^{2} \\
P & \longmapsto \tilde{P},
\end{aligned}
$$

defined by requiring that the three points $N, P, \tilde{P}$ are collinear. By a straightforward calculation it follows that the map $\pi_{S}$ is given explicitly by

$$
\pi_{\boldsymbol{S}}(x, y, z)=\frac{2 R}{2 R-z}(x, y) .
$$

\footnotetext{
${ }^{1}$ Here we are considering the Euclidean space equipped with Cartesian coordinates whose axis are labeled as $x, y, z$ according to the ordering induced by the canonical orthonormal basis of $\mathbb{R}^{3}$.
} 
Using the angles $\phi$ and $\theta$ as previously defined, a generic point $P=$ $(x, y, z)$ on the sphere in these coordinates has a local parameterization given by

$$
\left[\begin{array}{l}
x \\
y \\
z
\end{array}\right]=R\left[\begin{array}{c}
\sin \theta \cos \phi \\
\sin \theta \sin \phi \\
1-\cos \theta
\end{array}\right]=\boldsymbol{P}(\phi, \theta) .
$$

Of course the map is a diffeomorphism of class $\mathscr{C}^{\infty}$. In these coordinates the stereographic projection $\pi_{\boldsymbol{S}}$ is defined as:

$$
R\left[\begin{array}{c}
\sin \theta \cos \phi \\
\sin \theta \sin \phi \\
1-\cos \theta
\end{array}\right] \longmapsto \frac{2 R}{1+\cos \theta}\left[\begin{array}{c}
\sin \theta \cos \phi \\
\sin \theta \sin \phi
\end{array}\right] .
$$

We recall that if $M \subset \mathbb{R}^{3}$ is a portion of a regular surface represented in Cartesian local coordinates by the vector equation

$$
\boldsymbol{P}(u, v):=\mathbf{0}+x(u, v) \boldsymbol{i}+y(u, v) \boldsymbol{j}+z(u, v) \boldsymbol{k}
$$

then $d \boldsymbol{P}=\boldsymbol{P}_{u} d u+\boldsymbol{P}_{v} d v$ for $\boldsymbol{P}_{u}=\left(x_{u}, y_{u}, z_{u}\right)$ and $\boldsymbol{P}_{v}=\left(x_{v}, y_{v}, z_{v}\right)$ and hence the metric is given by $d s^{2}=\|d \boldsymbol{P}\|^{2}$. With the above parametrization of the sphere it follows that

$$
\boldsymbol{P}_{\theta}=R(\cos \theta \cos \phi, \cos \theta \sin \phi, \sin \theta), \quad \boldsymbol{P}_{\phi}=R(-\sin \theta \sin \phi, \sin \theta \cos \phi, 0) .
$$

Denoting by $\boldsymbol{g}$ and $\boldsymbol{g}_{\boldsymbol{S}}$ respectively the Riemannian metric on the sphere $\boldsymbol{S}$ and the metric on the plane induced by the stereographic projection, we have

$$
\boldsymbol{g}:=R^{2}\left[\begin{array}{ll}
\sin ^{2} \theta & 0 \\
0 & 1
\end{array}\right], \quad \boldsymbol{g}_{\boldsymbol{S}}:=\frac{4}{(1+\cos \theta)^{2}} \boldsymbol{g} .
$$

As a consequence of the above calculation the following result holds.

Lemma 3.1. The manifolds $(\boldsymbol{S}, \boldsymbol{g})$ and $\left(\overline{\mathbb{R}^{2}}, \boldsymbol{g}_{\boldsymbol{S}}\right)$ are in the same conformal class, where $\overline{\mathbb{R}^{2}}$ denotes the Alexandroff compactification of $\mathbb{R}^{2}$.

\section{Co-geodesic flows}

Let $\boldsymbol{G}$ and $\boldsymbol{G}_{\boldsymbol{S}}$ be the matrices corresponding to the inverse of $\boldsymbol{g}$ and $\boldsymbol{g}_{\boldsymbol{S}}$ respectively; thus we have

$$
\boldsymbol{G}=R^{-2}\left(\begin{array}{ll}
\sin ^{-2} \theta & 0 \\
0 & 1
\end{array}\right) \quad \text { and } \quad \boldsymbol{G}_{\boldsymbol{S}}=\frac{(1+\cos \theta)^{2}}{4} \boldsymbol{G} .
$$

We denote by $\hat{\boldsymbol{S}}=\boldsymbol{S} \backslash\{N, S\}$ the sphere minus the north and the south pole, and by $T^{*} \hat{\boldsymbol{S}}$ its cotangent bundle. On $\hat{\boldsymbol{S}}=\boldsymbol{S} \backslash\{N, S\}$ the following Hamiltonian function is well-defined:

$$
\begin{aligned}
H_{\text {free }}: T^{*} \hat{\boldsymbol{S}} & \rightarrow \mathbb{R} \\
(\boldsymbol{q} ; \boldsymbol{p}) & \mapsto \frac{1}{2}\langle\boldsymbol{G} \boldsymbol{p}, \boldsymbol{p}\rangle
\end{aligned}
$$

where $\boldsymbol{q}:=(\phi, \theta)$ are the positions and $\boldsymbol{p}:=\left(p_{\phi}, p_{\theta}\right)$ are the momenta. With this choice the Hamiltonian function is given by

$$
H_{\text {free }}\left(\phi, \theta ; p_{\phi}, p_{\theta}\right)=\frac{1}{2 R^{2}}\left(\frac{1}{\sin ^{2} \theta} p_{\phi}^{2}+p_{\theta}^{2}\right)
$$


and, as a particular case of (5), the co-geodesic flow on the sphere is given by

$$
\left\{\begin{array}{l}
\dot{\phi}=\frac{1}{R^{2} \sin ^{2} \theta} p_{\phi} \\
\dot{\theta}=\frac{p_{\theta}}{R^{2}} \\
\dot{p}_{\phi}=0 \\
\dot{p}_{\theta}=\frac{\cos \theta}{R^{2} \sin ^{3} \theta} p_{\phi}^{2}
\end{array} .\right.
$$

The co-geodesic flow on the plane $\{\boldsymbol{z}=0\}$ is equivalent to the above one through the stereographic projection. Since the metrics $\boldsymbol{g}$ and $\boldsymbol{g}_{\boldsymbol{S}}$ are in the same conformal class, the new system is easily derived using (4) with $\lambda=$ $\frac{(1+\cos \theta)^{2}}{4}$.

However, on the plane we prefer to use the Cartesian coordinates $(x, y)$ rather than the angular coordinates $(\phi, \theta)$. The latter are related to the former by the transformation

$$
\phi=\arctan \left(\frac{y}{x}\right), \quad \theta=2 \arctan \left(\frac{\sqrt{x^{2}+y^{2}}}{2 R}\right) .
$$

Hence, denoting by $\mathbb{R}_{s}^{2}$ the plane endowed with the metric $\boldsymbol{g}_{\boldsymbol{S}}$, the Hamiltonian function for the co-geodesic flow on $\left(\mathbb{R}^{2}, \boldsymbol{g}_{\boldsymbol{S}}\right)$

$$
K_{\text {free }}: T^{*} \mathbb{R}_{s}^{2} \rightarrow \mathbb{R}:(\boldsymbol{q} ; \boldsymbol{p}) \mapsto \frac{1}{2}\left\langle\boldsymbol{G}_{\boldsymbol{S}} \boldsymbol{p}, \boldsymbol{p}\right\rangle
$$

is explicitly given by

$$
K_{\text {free }}\left(x, y ; p_{x}, p_{y}\right)=\boldsymbol{l}(x, y)\left[\boldsymbol{a}(x, y) p_{x}^{2}+p_{y}^{2}\right]
$$

where $\boldsymbol{q}:=(x, y)$ are the positions and $\boldsymbol{p}:=\left(p_{x}, p_{y}\right)$ are the momenta and

$$
\boldsymbol{a}(x, y):=\left[\frac{4 R^{2}+x^{2}+y^{2}}{4 R \sqrt{x^{2}+y^{2}}}\right]^{2}, \quad l(x, y):=\frac{8 R^{2}}{\left(4 R^{2}+x^{2}+y^{2}\right)^{2}} .
$$

In order to derive these expressions we used the identities

$$
\sin \theta=\frac{4 R \sqrt{x^{2}+y^{2}}}{4 R^{2}+x^{2}+y^{2}}, \quad \sin \phi=\frac{y}{\sqrt{x^{2}+y^{2}}}
$$

and

$$
\frac{(1+\cos \theta)^{2}}{8 R^{2}}=\frac{8 R^{2}}{\left(4 R^{2}+x^{2}+y^{2}\right)^{2}}
$$

Note that $\boldsymbol{a}(x, y)$ corresponds to the term $(\sin \theta)^{-2}$, while $\boldsymbol{l}(x, y)$ is just $\frac{1}{2 R^{2}} \lambda$. To the Hamiltonian $K_{\text {free }}$ is associated the Hamiltonian flow

$$
\left\{\begin{array}{l}
\dot{x}=2(\boldsymbol{a} \boldsymbol{l})(x, y) p_{x} \\
\dot{y}=2 \boldsymbol{l}(x, y) p_{y} \\
\dot{p}_{x}=-\left[\partial_{x}(\boldsymbol{a} \boldsymbol{l}) p_{x}^{2}+\left(\partial_{x} \boldsymbol{l}\right) p_{y}^{2}\right] \\
\dot{p}_{y}=-\left[\partial_{y}(\boldsymbol{a} \boldsymbol{l}) p_{x}^{2}+\left(\partial_{y} \boldsymbol{l}\right) p_{y}^{2}\right]
\end{array}\right.
$$


where

$$
\begin{aligned}
(\boldsymbol{a} \boldsymbol{l})(x, y) & :=\frac{1}{2\left(x^{2}+y^{2}\right)}, \quad \partial_{x} \boldsymbol{l}(x, y)=-\frac{32 R^{2} x}{\left(4 R^{2}+x^{2}+y^{2}\right)^{3}} \\
\partial_{y} \boldsymbol{l}(x, y) & =-\frac{32 R^{2} y}{\left(4 R^{2}+x^{2}+y^{2}\right)^{3}} .
\end{aligned}
$$

\section{Position of the problem}

We are now in the position to introduce a conservative force field on the sphere that perturbs, not necessarily by small amounts, the dynamics of the free particle on the sphere. As already said in the Sect. 1, we are interested in the one center problem on the sphere, thus we introduce a force field pointing towards (or outwards) a fixed point of the surface.

For the reasons discussed at the end of the Sect. 1, we place the singularity of the potential at the point $Q=(0, R, R)$.

On $\boldsymbol{S} \backslash\{Q\}$ we define the

1. geodesic logarithmic potential as

$$
U_{\text {geo }}(P):=-\frac{\Gamma}{4 \pi} \log \left(d_{\boldsymbol{S}}(P, Q)\right)
$$

where $d_{\boldsymbol{S}}(P, Q)$ is the geodesic distance between $P$ and $Q$, that is the length of the shortest arc of a great circle passing through the two points $P$ and $Q$;

2. Euclidean logarithmic potential as

$$
U_{\text {euc }}(P):=-\frac{\Gamma}{4 \pi} \log (d(P, Q))
$$

where $d(P, Q)$ is the Euclidean distance between $P$ and $Q$, that is the chord in $\mathbb{R}^{3}$ joining the two points $P$ and $Q$.

Because the qualitative dynamics is mostly the same for the two cases, here we carefully analyze the geodesic case and we point out from time to time the differences with the Euclidean case, if any.

For any couple of points $P_{1}, P_{2} \in \boldsymbol{S} \subset \mathbb{R}^{3}$, the geodesic distance $d_{\boldsymbol{S}}\left(P_{1}\right.$, $\left.P_{2}\right)$ is given by

$$
d_{\boldsymbol{S}}(P, Q):=R \arccos \frac{\left\langle P_{1}-C, P_{2}-C\right\rangle}{R^{2}},
$$

where $\langle\cdot, \cdot\rangle$ is the Euclidean scalar product in $\mathbb{R}^{3}$. Thus, in the $(\phi, \theta)$-coordinates, the geodesic distance between any point $P \in \boldsymbol{S}$ and the vortex point $Q$ is

$$
d_{\boldsymbol{S}}(P, Q)=R \arccos (\sin \phi \sin \theta) .
$$

We augment the free-motion Hamiltonian $H_{\text {free }}$ with the above potential function and we define

$$
H_{g e o}: T^{*}(\hat{\boldsymbol{S}} \backslash\{Q\}) \rightarrow \mathbb{R}
$$




$$
H_{\text {geo }}\left(\phi, \theta, p_{\phi}, p_{\theta}\right):=H_{\text {free }}\left(\phi, \theta, p_{\phi}, p_{\theta}\right)+\frac{\Gamma}{4 \pi} \log (R \arccos (\sin \phi \sin \theta)) .
$$

The last is the Hamiltonian that governs the motion of a particle constrained to move on a sphere and subjected to the force field $\boldsymbol{f}(P)=$ $\nabla U_{\text {geo }}(P)$ : at any point $P \neq Q$ the force points towards $Q$ if $\Gamma>0$ or in the opposite direction otherwise, and it is proportional to the inverse of the distance $d_{\boldsymbol{S}}(P, Q)$.

On $\left(\mathbb{R}^{2}, \boldsymbol{g}_{\boldsymbol{S}}\right)$, using the relations $(9)$, the distance $d_{\boldsymbol{S}}(P, Q)$ becomes the function

$$
\boldsymbol{b}(x, y):=R \arccos \left(\frac{4 R y}{4 R^{2}+x^{2}+y^{2}}\right)=R \arccos \left(1-\frac{x^{2}+(y-2 R)^{2}}{4 R^{2}+x^{2}+y^{2}}\right)
$$

therefore we introduce the Hamiltonian

$$
\begin{gathered}
K_{\text {geo }}: T^{*}\left(\mathbb{R}_{s}^{2} \backslash\{0, V\}\right) \rightarrow \mathbb{R} \\
K_{\text {geo }}\left(x, y, p_{x}, p_{y}\right):=K_{\text {free }}\left(x, y, p_{x}, p_{y}\right)+\frac{\Gamma}{4 \pi} \log \boldsymbol{b}(x, y) .
\end{gathered}
$$

We observe that $\log \boldsymbol{b} \in \mathscr{C}\left(\mathbb{R}^{2} \backslash\{(0,2 R)\}\right)$ and $\log \boldsymbol{b} \in \mathscr{C}^{1}\left(\mathbb{R}^{2} \backslash\{(0,2 R)\right.$, $(0,-2 R)\})$ : indeed the point $V=(0,2 R)$ is the stereographic projection of the vortex $Q \in S$, point of singularity of the potential, while the point $V^{\prime}=(0,-2 R)$ corresponds to the point $Q^{\prime}$ on the sphere antipodal to the vortex point. Here the potential is continuous but not differentiable, and this reflects the fact that in the antipodal point the force field is not determined, being the geodesics arc joining $Q^{\prime}$ to $Q$ not uniquely defined. In the following, anytime we refer to "the singularity" or to "the singular point" of the system we mean the singularity placed in the vortex point $V$ (or $Q$ if seen on the sphere), due to the logarithmic potential.

Hamilton's equations associated to $K_{\text {geo }}$ can be written as follows:

$$
\left\{\begin{array}{l}
\dot{x}=2(\boldsymbol{a} \boldsymbol{l})(x, y) p_{x} \\
\dot{y}=2 \boldsymbol{l}(x, y) p_{y} \\
\dot{p}_{x}=-\left[\partial_{x}(\boldsymbol{a l}) p_{x}^{2}+\left(\partial_{x} \boldsymbol{l}\right) p_{y}^{2}+\Gamma /(4 \pi) \boldsymbol{b}(x, y)^{-1} \partial_{x} \boldsymbol{b}(x, y)\right] \\
\dot{p}_{y}=-\left[\partial_{y}(\boldsymbol{a} \boldsymbol{l}) p_{x}^{2}+\left(\partial_{y} \boldsymbol{l}\right) p_{y}^{2}+\Gamma /(4 \pi) \boldsymbol{b}(x, y)^{-1} \partial_{y} \boldsymbol{b}(x, y)\right]
\end{array} .\right.
$$

For the Euclidean logarithmic potential the Hamiltonian is:

$$
H_{\text {euc }}\left(\phi, \theta, p_{\phi}, p_{\theta}\right):=H_{\text {free }}\left(\phi, \theta, p_{\phi}, p_{\theta}\right)+\frac{\Gamma}{8 \pi} \log \left(2 R^{2}(1-\sin \theta \sin \phi)\right)
$$

being $2 R^{2}(1-\sin \theta \sin \phi)=\|\overline{P Q}\|^{2}$. Hamilton's equations on the space $\left(\mathbb{R}^{2}, \boldsymbol{g}_{\boldsymbol{S}}\right)$ are still given by (14), once the function $\boldsymbol{b}$ has been replaced by

$$
\boldsymbol{b}_{\text {euc }}(x, y):=\left(\frac{2 R^{2}\left[x^{2}+(y-2 R)^{2}\right]}{4 R^{2}+x^{2}+y^{2}}\right)^{\frac{1}{2}} .
$$

A remarkable difference between the geodesic and Euclidean case is that the function $\log \boldsymbol{b}_{e u c}$ is continuous and differentiable in the antipodal point $V^{\prime}$. 


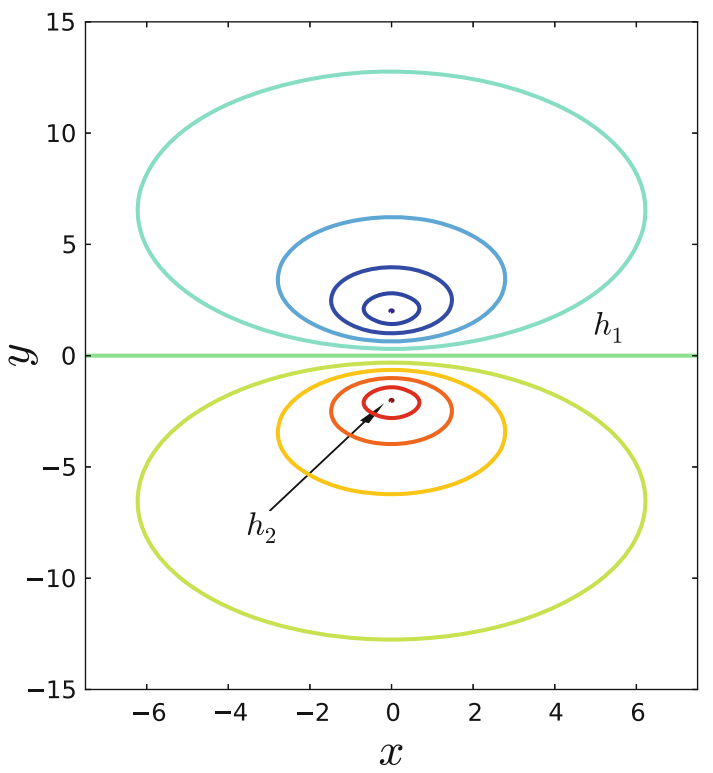

FiguRE 3. Zero-level curves of the function $\tilde{E}_{h}(x, y)$ in the geodesic case, for $\Gamma=R=1$ and $h=\log (n \pi / 10) /(4 \pi), n=$ $0, \ldots 10$. For $n=10$ the forbidden region is just the antipodal point at $(0,-2)$; for $n=0$ the allowed region reduces to the vortex point at $(0,2)$. The euclidean case is analogous, with shifted energy values

Indeed it results $\nabla \log \boldsymbol{b}_{\text {euc }}\left(V^{\prime}\right)=0$ and this agrees with the physical interpretation that the force field generated by $U_{\text {euc }}$ at the point $P \neq Q$ is proportional to the component of the chord $P Q$ tangent to the sphere.

\section{Energy hypersurfaces, regularization and flow}

We begin the analysis of system (14) with the description of the topology of the constant-energy hypersurfaces associated to $K_{\text {geo }}$. For any $h \in \mathbb{R}$ the hypersurface of constant energy $h$ is given by

$$
\begin{aligned}
\widetilde{\Sigma}_{h}:= & \left\{\left(x, y, p_{x}, p_{y}\right) \in T^{*} X: K_{\text {mech }}\left(x, y, p_{x}, p_{y}\right)=h\right\} \\
= & \left\{\left(x, y, p_{x}, p_{y}\right) \in T^{*} X: \boldsymbol{a}(x, y) p_{x}^{2}+p_{y}^{2}=\frac{1}{\boldsymbol{l}(x, y)}\right. \\
& \times(h-\Gamma /(4 \pi) \log (\boldsymbol{b}(x, y)))\}
\end{aligned}
$$

where $X:=\mathbb{R}^{2} \backslash\{0, V\}$ denotes the configuration space and $T^{*} X$ the phase space (the cotangent bundle over $X$ ).

Since the function $\boldsymbol{a}(x, y)$ is strictly positive, for any value of $h$ the motion is allowed only in the region of the configuration space where the right hand 
side of the equation in the definition of $\widetilde{\Sigma}_{h}$ is positive (Fig. 3). In the Lemma 5.1 below, the analysis is performed for $\Gamma>0$ : changing the sign of $\Gamma$ simply switches the allowed region with the forbidden region.

Lemma 5.1. For any fixed $h$ let $\widetilde{E}_{h}: \mathbb{R}^{2} \rightarrow \mathbb{R}$ be defined by:

$$
\widetilde{E}_{h}(x, y):=(h-\Gamma /(4 \pi) \log (\boldsymbol{b}(x, y))),
$$

and let $h_{1}=\frac{\Gamma}{4 \pi} \log \left(\frac{\pi}{2} R\right), h_{2}=\frac{\Gamma}{4 \pi} \log (\pi R)$. Then

1. for every $h>h_{2}$ the surface $\widetilde{E}_{h}(x, y)$ is positive for any $(x, y) \in \mathbb{R}^{2}$;

2. for any $h \in\left(h_{1}, h_{2}\right)$ there exists a disk $D_{h}^{1}$ in the $\{y<0\}$ half-plane and containing the point $(0,-2 R)$ such that $\widetilde{E}_{h}(x, y)$ is positive for each $(x, y) \in \mathbb{R}^{2} \backslash D_{h}^{1}$ and negative otherwise;

3. for any $h<h_{1}$ there exists a disk $D_{h}^{2}$ in the $\{y>0\}$ half-plane, containing the point $(0,2 R)$, such that $\widetilde{E}_{h}(x, y)$ is positive for all $(x, y) \in D_{h}^{2}$ and negative otherwise.

Proof. The logarithm and the arccos are monotone functions, therefore the level sets of $\widetilde{E}_{h}(x, y)$ corresponds to the level sets of $\frac{x^{2}+(y-2 R)^{2}}{4 R^{2}+x^{2}+y^{2}}$. For any $\delta \in[0,2]$ the $\delta$-level set is the circle $C_{\delta}$ with equation

$$
x^{2}+y^{2}-\frac{4 R}{1-\delta} y+4 R^{2}=0 .
$$

The result of the lemma follows from a straightforward analysis of $C_{\delta}$ and by computing the value of $\tilde{E}_{h}(x, y)$ in correspondence of $\delta=2$ and $\delta=0$. In particular $C_{\delta}$ collapses to the points $(0,2 R)$ and $(0,-2 R)$ respectively at $\delta=0$ and $\delta=2$ and degenerates to the $x$-axis for $\delta=1$.

Remark 5.2. In case of the Euclidean potential the results of Lemma 5.1 hold with $h_{1}=\frac{\Gamma}{8 \pi} \log \left(2 R^{2}\right)$ and $h_{2}=\frac{\Gamma}{4 \pi} \log (2 R)$.

We now introduce the space and time coordinate transformations, analogous to those of McGehee, that allow for the removal of the logarithmic singularity in the system (14). Let us define the functions

$$
\left\{\begin{array}{l}
\varphi_{1}(r):=r e^{-1 / r^{2}} \\
\varphi_{2}(r):=1 / r
\end{array}\right.
$$

Following the notation of [18], we introduce the change of variables

$$
\left\{\begin{array}{l}
x=\varphi_{1}(r) s_{1} \\
y=\varphi_{1}(r) s_{2}+2 R
\end{array}, \quad\left\{\begin{array}{l}
p_{x}=\varphi_{2}(r) z_{x} \\
p_{y}=\varphi_{2}(r) z_{y}
\end{array}\right.\right.
$$

where $s=\left(s_{1}, s_{2}\right)=(\cos \alpha, \sin \alpha) \in \mathbb{S}^{1}$ is a point on the unit circle. In the new coordinates $(r, \alpha)$, the functions $\boldsymbol{a}(x, y), \boldsymbol{l}(x, y), \boldsymbol{a} \boldsymbol{l}(x, y)$ and $\boldsymbol{b}(x, y)$ introduced in (8) and (13) become 


$$
\begin{aligned}
& \boldsymbol{a}(r, \alpha)=\left[\frac{8 R^{2}+4 R \varphi_{1}(r) s_{2}+\varphi_{1}^{2}}{4 R \sqrt{\varphi_{1}^{2}+4 R^{2}+4 R \varphi_{1}(r) s_{2}}}\right]^{2}, \\
& \boldsymbol{b}(r, \alpha)=R \arccos \left(1-\frac{\varphi_{1}(r)^{2}}{8 R^{2}+\varphi_{1}(r)^{2}+4 R \varphi_{1}(r) s_{2}}\right), \\
& \boldsymbol{l}(r, \alpha)=\frac{8 R^{2}}{\left(4 R^{2}+\varphi_{1}^{2}+4 R \varphi_{1} s_{2}\right)^{2}}, \quad \text { and } \\
& (\boldsymbol{a l})(r, \alpha)=\frac{1}{2\left(4 R^{2}+\varphi_{1}^{2}+4 R \varphi_{1} s_{2}\right)}
\end{aligned}
$$

and the energy surfaces $\Sigma_{h}$ can be written as

$$
\begin{gathered}
\Sigma_{h}=\left\{\left(r, \alpha, z_{x}, z_{y}\right) \in \mathbb{R}^{+} \times \mathbb{S}^{1} \times \mathbb{R}^{2}: \boldsymbol{a}(r, \alpha) z_{x}^{2}+z_{y}^{2}\right. \\
=\frac{r^{2}}{\boldsymbol{l}(r, \alpha)}(h-\Gamma /(4 \pi) \log (\boldsymbol{b}(r, \alpha))\} .
\end{gathered}
$$

We also observe that

- $\lim _{r \rightarrow 0^{+}} \boldsymbol{a}(r, \alpha)=1 \quad$ uniformly with respect to $\alpha$;

- $\lim _{r \rightarrow 0^{+}} \boldsymbol{b}(r, \alpha)=0 \quad$ uniformly with respect to $\alpha$;

- $\lim _{r \rightarrow 0^{+}} \boldsymbol{l}(r, \alpha)=1 /\left(2 R^{2}\right) \quad$ uniformly with respect to $\alpha$.

By taking into account the definition of the functions $\varphi_{j}$, the right hand side of the equation defining the level set $\Sigma_{h}$ reduces to

$$
\begin{aligned}
& \hat{E}(h, r, s):=\frac{r^{2}}{l(r, s)} \\
& \quad \times\left[h-\frac{\Gamma}{4 \pi} \log \left(R \arccos \left(1-\frac{r^{2} e^{-2 / r^{2}}}{8 R^{2}+r^{2} e^{-2 / r^{2}}+4 R r e^{-1 / r} s_{2}}\right)\right)\right] .
\end{aligned}
$$

As it was already implicit in Lemma 5.1 , in the attractive case $(\Gamma>0)$ the vortex point $V$ lies in the allowed region of every energy level $h$ and the value of $\tilde{E}_{h}(x, y)$ diverges to infinity as $(x, y) \rightarrow V$. However, a first important consequence of this change of variables is that in the coordinates $(r, s)$ the kinetic energy remains bounded when a collision occurs. Indeed it holds

\section{Lemma 5.3.}

$$
\lim _{r \rightarrow 0^{+}} \hat{E}(h, r, s)=\frac{\Gamma R^{2}}{2 \pi}
$$

Proof. By taking into account the asymptotic behavior of $\boldsymbol{l}(r, \alpha)$ for $r \rightarrow 0$, we get

$$
\begin{aligned}
\lim _{r \rightarrow 0^{+}} \hat{E}(h, r, s) & =-2 R^{2} \frac{\Gamma}{4 \pi} \lim _{r \rightarrow 0^{+}} r \log \left(R \arccos \left(1-\frac{r e^{-2 / r}}{8 R^{2}}\right)\right) \\
& =\frac{\Gamma}{2 \pi} \lim _{r \rightarrow 0^{+}} \frac{r(2+r) e^{-2 / r}}{\arccos \left(1-\frac{r e^{-2 / r}}{8 R^{2}}\right) \sqrt{1-\left(1-\frac{r e^{-2 / r}}{8 R^{2}}\right)^{2}}} .
\end{aligned}
$$




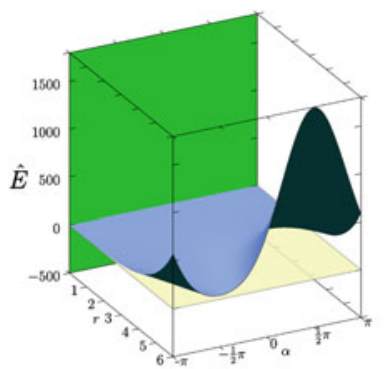

(a)

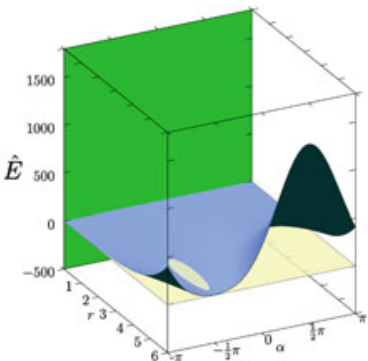

(b)

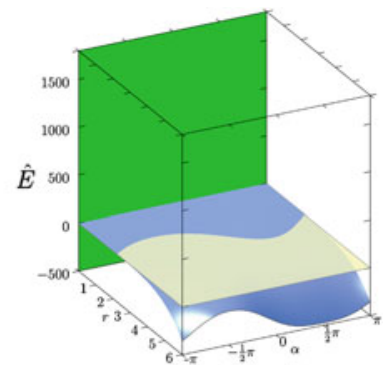

(c)

Figure 4. Graph of the function $\hat{E}$ in the three cases: a $h>$ $h_{2}, \mathbf{b} h_{1} \leq h \leq h_{2}$, (c) $h<h_{1}$. The intersection between the surface and the plane $\hat{E}=0$ is the zero-velocity manifold. The figure was drawn using the geodetic distance. The euclidean case is qualitatively the same

The result follows by the fact that $\sqrt{1-(1-t)^{2}} \arccos (1-t)=2 t+o(t)$.

From now on, we shall only consider the attractive case. Thus we assume

$$
\Gamma>0 \text {. }
$$

The intersection between one (and hence every) energy hypersurface $\Sigma_{h}$ with $r=0$ is called total collision manifold. In virtue of the limit (19), we may conclude that

- the total collision manifold does not depend on the fixed energy level $h$; otherwise stated it is a boundary for every constant-energy surface;

- it is diffeomorphic to the two dimensional torus $\mathbb{T}:=\mathbb{S}^{1} \times \mathbb{S}^{1}$.

From the dynamical viewpoint an important role is played by the zero set of the function $\hat{E}$ :

$$
Z_{h}:=\{(r, \alpha) \in X: \hat{E}(h, r, \alpha)=0\} .
$$

In the following we refer to this set as the zero velocity manifold in $\Sigma_{h}$. Rephrasing the results of Lemma 5.1 in terms of the new coordinates $(r, \alpha)$ it readily follows that $\boldsymbol{Z}_{h}$ is empty in the first case $\left(h>h_{2}\right)$ and non-empty otherwise. In the second case, $\left(h_{1} \leq h \leq h_{2}\right)$, the zero velocity manifold is represented by a simple closed curve homeomorphic to a circle (or to a point in the limit $h \rightarrow h_{2}$ ). The motion is forbidden inside the region bounded by the curve. In the third case, the zero set can be seen as the graph of a single-valued function $\alpha \mapsto r(\alpha)$ and the function $\hat{E}$ is positive for $0<r<r(\alpha)$ (Fig. 4).

\section{Regularization and McGehee coordinates}

We now rewrite the equations of motion (14) in the new system of coordinates $r, \alpha$ and $\boldsymbol{z}$. In order to preserve the continuity of the flow with respect to the initial data, we need to ensure that the transformed system has an everywhere differentiable vector field. To this purpose we rescale the time variable in terms of the distance from the singularity with the effect to exponentially decrease 
the velocities near the singularity. As a result the collision solutions (which are singular in the old coordinates) move along smooth orbits that asymptotically converge to the collision manifold.

Let us define $d \tau=\varphi_{2}(r) \varphi_{1}^{-1}(r) d t$ and use the notation

$$
\langle\boldsymbol{z}, \boldsymbol{s}(\alpha)\rangle_{a}:=\boldsymbol{a}(x, y) z_{x} \cos \alpha+z_{y} \sin \alpha .
$$

With the help of the identities

$$
\frac{\varphi_{1}(r)}{\varphi_{1}^{\prime}(r)}=\frac{r^{3}}{r^{2}+2}, \quad \frac{\varphi_{1}(r)}{\varphi_{2}^{2}(r)}=r^{3} e^{-1 / r^{2}}, \quad \frac{\varphi_{1}(r) \varphi_{2}^{\prime}(r)}{\varphi_{2}(r) \varphi_{1}^{\prime}(r)}=-\frac{r^{2}}{r^{2}+2}
$$

the Hamiltonian equations in (14) become

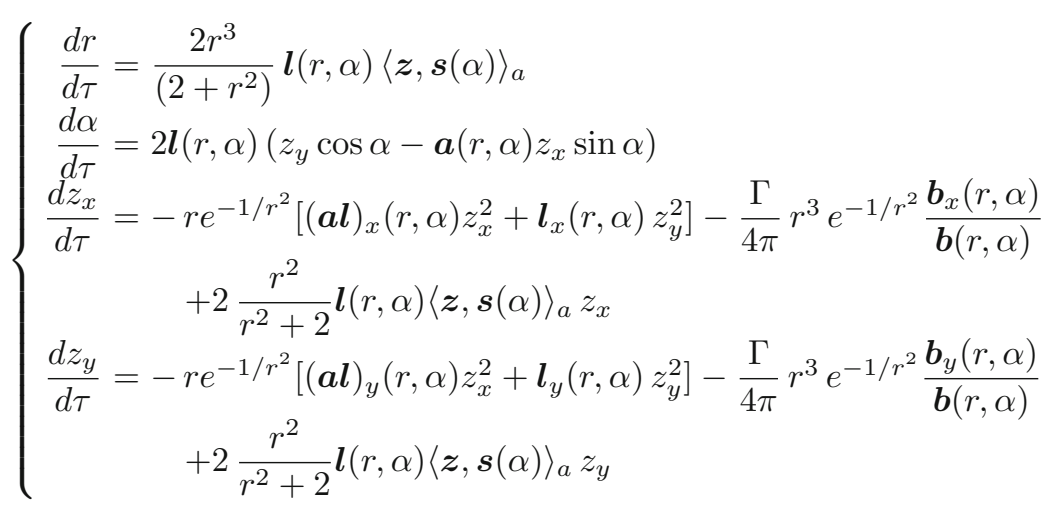

where the subscripts in $(\boldsymbol{a l})_{x}(r, \boldsymbol{s}),(\boldsymbol{a l})_{y}(r, \boldsymbol{s})\left(\operatorname{resp} . \boldsymbol{b}_{x}(r, \boldsymbol{s}), \boldsymbol{b}_{y}(r, \boldsymbol{s})\right)$ denote the partial derivative with respect to the old Cartesian variables. The derivative is then evaluated in the new coordinates at the point $(r, \alpha)$. These equations are no longer singular at $r=0$ : in fact,

$$
\begin{aligned}
& {\left[\frac{\frac{\partial_{x} \boldsymbol{b}}{\boldsymbol{b}_{\boldsymbol{b}}}}{\frac{\partial_{y} \boldsymbol{b}}{\boldsymbol{b}}}\right](r, \alpha)=\frac{1}{\arccos \left(1-\frac{\varphi_{1}(r)^{2}}{C(r, \alpha)}\right) \sqrt{1-\left(1-\frac{\varphi_{1}(r)^{2}}{C(r, \alpha)}\right)^{2}} C(r, \alpha)^{2}}} \\
& \times\left[\begin{array}{l}
8 R \varphi_{1}(r) \cos \alpha\left(\varphi_{1}(r) \sin \alpha+2 R\right) \\
4 R\left(\varphi_{1}^{2}(r)\left(\sin ^{2} \alpha-\cos ^{2} \alpha\right)-4 R \varphi_{1}(r) \sin \alpha\right)
\end{array}\right]
\end{aligned}
$$

where $C(r, \alpha)=8 R^{2}+\varphi_{1}^{2}(r)+4 R \varphi_{1}(r) \sin \alpha$, and it follows

$$
\left[\begin{array}{c}
\frac{\partial_{x} \boldsymbol{b}}{\boldsymbol{b}} \\
\frac{\partial_{y} \boldsymbol{b}}{\boldsymbol{b}}
\end{array}\right](r, \alpha) \rightarrow \varphi_{1}^{-1}\left[\begin{array}{c}
\cos \alpha \\
\sin \alpha
\end{array}\right] .
$$

Thus the time change produces the effect to regularize the singularity. In addition the $\{r=0\}$ manifold results to be invariant.

From a naive point of view, the study of the flow on the collision manifold could appear meaningless, since the manifold is the image of just a singular point where the orbits cease to exists. In reality, the properties of the flow 
on such manifold yield information on the behavior of the orbits close to the singularity.

In order to simplify the system (21), we introduce a further change of coordinates and time rescaling. Using the energy relation

$$
\boldsymbol{a}(r, \alpha) z_{x}^{2}+z_{y}^{2}=\hat{E}(h, r, \alpha),
$$

let us define $\psi$ and $\sigma$ such that

$$
\left\{\begin{array}{rl}
z_{x} & =\sqrt{\hat{E}(h, r, \alpha) / \boldsymbol{a}(r, \alpha)} \cos \psi \\
z_{y} & =\sqrt{\hat{E}(h, r, \alpha)} \sin \psi \\
d \tau & =\sqrt{\hat{E}(h, r, \alpha)} d \sigma
\end{array} .\right.
$$

Denote by $A_{1}(r, \alpha, z), A_{2}(r, \alpha, z)$ the right hand side of, respectively, the third and fourth equation in (21), so that $\frac{d z_{x}}{d \tau}=A_{1}, \frac{d z_{y}}{d \tau}=A_{2}$. Therefore, on any fixed-energy shell, the system given in (21) reduces to

$$
\left\{\begin{array}{l}
\frac{d r}{d \sigma}=\frac{2 r^{3} \hat{E}(h, r, \alpha)}{\left(2+r^{2}\right)} \boldsymbol{l}(r, \alpha)(\sqrt{\boldsymbol{a}(r, \alpha)} \cos \psi \cos \alpha+\sin \psi \sin \alpha) \\
\frac{d \alpha}{d \sigma}=2 \hat{E}(h, r, \alpha) \boldsymbol{l}(r, \alpha)(\sin \psi \cos \alpha-\sqrt{\boldsymbol{a}(r, \alpha)} \cos \psi \sin \alpha) \\
\frac{d \psi}{d \sigma}=B(r, \alpha, \psi)
\end{array}\right.
$$

where $B$ is given by:

$$
\begin{aligned}
B(r, \alpha, \psi):= & -\sqrt{\boldsymbol{a}} \sin \psi A_{1}+\sqrt{\boldsymbol{a}} \sin \psi \cos \psi \frac{d}{d \tau}\left(\sqrt{\frac{\hat{E}}{\boldsymbol{a}}}\right) \\
& +A_{2} \cos \psi-\frac{d}{d \tau}(\sqrt{\hat{\hat{E}}}) \sin \psi \cos \psi
\end{aligned}
$$

\section{Flow and invariant manifolds}

Recalling that $\boldsymbol{l}$ is everywhere positive, the rest points of (26) correspond to solutions of the following systems:

$$
\left\{\begin{array} { l } 
{ r = 0 } \\
{ f _ { 2 } ( r , \alpha , \psi ) = 0 } \\
{ B ( r , \alpha , \psi ) = 0 }
\end{array} \quad \text { or } \quad \left\{\begin{array} { l } 
{ f _ { 1 } ( r , \alpha , \psi ) = 0 } \\
{ f _ { 2 } ( r , \alpha , \psi ) = 0 } \\
{ B ( r , \alpha , \psi ) = 0 }
\end{array} \quad \text { or } \quad \left\{\begin{array}{l}
\hat{E}(h, r, \alpha)=0 \\
B(r, \alpha, \psi)=0
\end{array}\right.\right.\right.
$$

where

$$
\begin{aligned}
& f_{1}(r, \alpha, \psi):=\sqrt{\boldsymbol{a}} \cos \psi \cos \alpha+\sin \psi \sin \alpha, \\
& f_{2}(r, \alpha, \psi):=\sin \psi \cos \alpha-\sqrt{\boldsymbol{a}} \cos \psi \sin \alpha .
\end{aligned}
$$

We immediately discard the second system as it does not allow any solution. In fact the first two equations are incompatibles. We note that $\boldsymbol{a} \rightarrow 1$ for $r \rightarrow 0$, 
thus the first system reduces to

$$
\left\{\begin{array}{l}
r=0 \\
\sin (\psi-\alpha)=0 \\
B(r, \alpha, \psi)=0
\end{array}\right.
$$

whose solutions correspond to fixed points on the collision manifold. The existence of solutions of the last system depends on the energy level $h$ : if $h \geq h_{2}$ the zero set of $\hat{E}$ is empty and no solutions exist. For $h \leq h_{2}$, solutions may exist.

Summarizing, any rest point either lies on the collision manifold or on the zero velocity manifold. Let us first consider the collision manifold: the asymptotic analysis of the function $B(r, \alpha, \psi)$ on the collision manifold (see Appendix A) gives

$$
\lim _{r \rightarrow 0^{+}} B(r, \alpha, \psi)=0
$$

It follows that

Lemma 5.4. The equilibria of the vector field given in (26) lying on the total collision manifold consist of two curves. In local coordinates $(r, \alpha, \psi)$ these curves are given by

(i) $\mathscr{P}_{1} \equiv(0, \alpha, \alpha)$;

(ii) $\mathscr{P}_{2} \equiv(0, \alpha, \pi+\alpha)$.

Proposition 5.5. For each $\alpha$, the equilibrium points

$$
(0, \alpha, \alpha) \in \mathscr{P}_{1}
$$

and

$$
(0, \alpha, \pi+\alpha) \in \mathscr{P}_{2}
$$

are degenerate saddles.

1. $\operatorname{dim} W^{u}\left(\mathscr{P}_{1}\right)=1, \quad \operatorname{dim} W^{s}\left(\mathscr{P}_{1}\right)=1, \quad \operatorname{dim} W^{0}\left(\mathscr{P}_{1}\right)=1$.

2. $\operatorname{dim} W^{u}\left(\mathscr{P}_{2}\right)=1, \quad \operatorname{dim} W^{s}\left(\mathscr{P}_{2}\right)=1, \quad \operatorname{dim} W^{0}\left(\mathscr{P}_{2}\right)=1$.

Proof. The flow on the collision manifold is given by

$$
\left\{\begin{array}{l}
\frac{d \alpha}{d \sigma}=\frac{\Gamma}{4 \pi} \sin (\psi-\alpha) \\
\frac{d \psi}{d \sigma}=0
\end{array}\right.
$$

whose orbits are parallel to $\alpha$-axis and flow from $\mathscr{P}_{2}$ to $\mathscr{P}_{1}$. The stability of the rest points is determined by the eigenvalues of the Jacobian matrix of (26). It follows (see Appendix A) that for any point $P_{1} \in \mathscr{P}_{1}$ and $P_{2} \in \mathscr{P}_{2}$ the eigenvalues are

$$
P_{1} \in \mathscr{P}_{1} \Rightarrow\left\{\begin{array}{l}
\lambda_{r}=0 \\
\lambda_{\alpha}=-\frac{\Gamma}{4 \pi}, \quad P_{2} \in \mathscr{P}_{2} \Rightarrow\left\{\begin{array}{l}
\lambda_{r}=0 \\
\lambda_{\psi}=0
\end{array} \lambda_{\alpha}=\frac{\Gamma}{4 \pi}\right. \\
\lambda_{\psi}=0
\end{array}\right.
$$




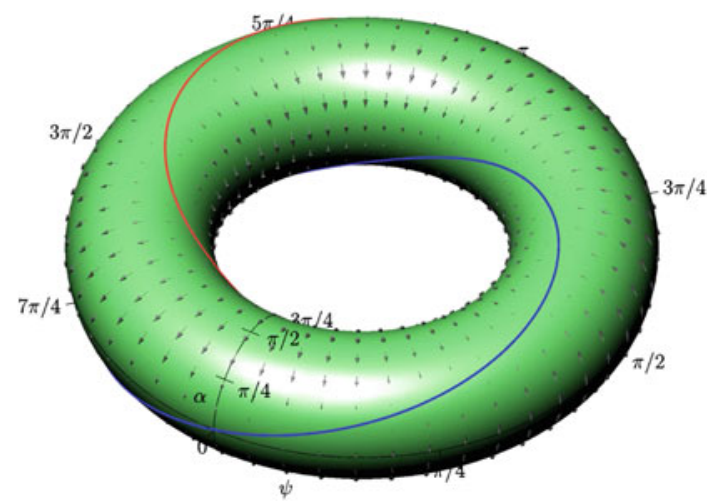

FiguRE 5. The collision manifold, the curves of rest points $\mathscr{P}_{1}$ (blue) and $\mathscr{P}_{2}($ red $)$, and the vector field of equations (28) (color figure online)

TABLE 1. Dimensions of the invariant manifolds along the equilibrium curves $\mathscr{P}_{1}$ and $\mathscr{P}_{2}$

\begin{tabular}{llll}
\hline & $\operatorname{dim} W^{s}$ & $\operatorname{dim} W^{u}$ & $\operatorname{dim} W^{0}$ \\
\hline At $\mathscr{P}_{1}$ & 1 & 1 & 1 \\
At $\mathscr{P}_{2}$ & 1 & 1 & 1 \\
\hline
\end{tabular}

The eigenvalues are coherent with the dynamics restricted on the collision manifold as given by (28), where $\mathscr{P}_{1}$ is an attractor and $\mathscr{P}_{2}$ is a repeller (Fig. 5). However, the presence of null eigenvalues implies that the linear approximation of the flow, taken alone, does not provide enough information to determine the qualitative dynamics close to the equilibrium points. Since the flow in the $\psi$ direction is null (and in fact $\psi$ can be regarded as the parameter which identifies an individual rest point along the curves $\mathscr{P}_{1}$ and $\mathscr{P}_{2}$ ), in order to determine the asymptotic behavior close to $P_{1}$ and $P_{2}$, it is enough to investigate the dynamics restricted to the $(r, \alpha)$-plane.

Definition 5.6. We shall say that the flow on the collision manifold is totally degenerate if the unstable manifold of an equilibrium point $\boldsymbol{P}_{1} \in \mathscr{P}_{1}$ coincides with the stable manifold of some equilibrium point $\boldsymbol{P}_{2} \in \mathscr{P}_{2}$.

Lemma 5.7. The flow on the total collision manifold is totally degenerate. More precisely

(i) $W^{u}\left(\boldsymbol{P}_{1}\right) \equiv W^{s}\left(\boldsymbol{P}_{2}\right)$;

(ii) $W^{u}\left(\boldsymbol{P}_{2}\right) \equiv W^{s}\left(\boldsymbol{P}_{1}\right)$;

where $\boldsymbol{P}_{1} \in \mathscr{P}_{1}$ and $\boldsymbol{P}_{2} \in \mathscr{P}_{2}$ are chosen in such a way that the last coordinate of the two points agrees.

Proof. The proof of this result follows by a straightforward integration of the equations of motion on the total collision manifold $r=0$. We omit the details and we refer to the equivalent proof of Lemma 7.4 in [18]. 
A direct consequence of the previous result is the following:

Corollary 5.8. (Existence of heteroclinic connections) There exists an heteroclinic connection between each equilibrium point $\boldsymbol{P}_{1} \in \mathscr{P}_{1}$ and the point $\boldsymbol{P}_{2} \in$ $\mathscr{P}_{2}$ where $\boldsymbol{P}_{1}, \boldsymbol{P}_{2}$ are chosen in such a way that they have the same projection on the first and third coordinate.

Proof. The proof of this result follows immediately by the previous result. By the fact that $r=0$ and $\psi$ is constant, it follows that the non equilibrium solutions are in the $(\alpha, \psi)$-plane lines parallel to the $\alpha$-axis. Moreover each point of equilibrium on $\mathscr{P}_{1}$ is attracting while each equilibrium point on $\mathscr{P}_{2}$ is repelling.

Moving out from the collision manifold, the two lines $\mathscr{P}_{1}$ and $\mathscr{P}_{2}$ exhibit the opposite stability character: indeed $\frac{d r}{d \sigma}>0$ for $\psi=\alpha$ and $\frac{d r}{d \sigma}<0$ when $\psi=\alpha+\pi$, meaning that the system goes into the collision along $\mathscr{P}_{2}$ and escapes from the collision along $\mathscr{P}_{1}$.

Next we examine the restpoints and the flow on the zero velocity manifold. This is more easily accomplished by looking at the system given in (21). Restpoints, in fact, are not changed by a time scaling. Setting $\hat{E}=0$ in the energy relation (24), which implies $z_{x}=z_{y}=0$, it follows that on the zero velocity manifold the dynamical system (21) reduces to:

$$
\left\{\begin{array}{l}
\frac{d r}{d \tau}=0 \\
\frac{d \alpha}{d \tau}=0 \\
\frac{d z_{x}}{d \tau}=-\frac{\Gamma}{4 \pi} r^{3} e^{-1 / r^{2}} \frac{\boldsymbol{b}_{x}(r, \alpha)}{\boldsymbol{b}(r, \alpha)} \\
\frac{d z_{y}}{d \tau}=-\frac{\Gamma}{4 \pi} r^{3} e^{-1 / r^{2}} \frac{\boldsymbol{b}_{y}(r, \alpha)}{\boldsymbol{b}(r, \alpha)}
\end{array} .\right.
$$

The restpoints on the zero velocity manifold (if any) correspond to the solutions of the equations:

$$
\frac{\boldsymbol{b}_{x}(r, \alpha)}{\boldsymbol{b}(r, \alpha)}=\frac{\boldsymbol{b}_{y}(r, \alpha)}{\boldsymbol{b}(r, \alpha)}=0 .
$$

An elementary calculation shows the following result.

Lemma 5.9. In the case of Euclidean logarithmic potential, for $h=h_{2}=$ $\frac{\Gamma}{4 \pi} \log (2 R)$ there exists only one restpoint on the zero velocity manifolds at $P:=\left(r_{*}, 3 \pi / 2\right)$ for $\varphi_{1}\left(r_{*}\right)=4 R$. For $h \neq h_{2}$ there are no restpoints.

Rephrased on the sphere, the rest point corresponds to the equilibrium solution of the dynamical system given by the initial data placed in the antipodal point with zero velocity.

As already noticed, in the case of the geodesic logarithmic potential the force field is not defined in the antipodal point, being the potential not differentiable. Therefore in this case there are no rest points on the zero velocity manifolds. 


\section{Global flow and dynamics on the sphere}

It is now possible to bring back on the sphere the results found on the stereographic plane in the previous sections.

The first concerns the flow around the singularity and the possible extension of the singular trajectories. Recalling the definition of transmission solution (Definition 1.1) we can now answer the main question and give the proof of Theorem 1.1.

Proof of Theorem 1.1. It follows from the regularization of the flow performed in the previous section and from the existence of the heteroclinic connections anywhere on the total collision manifolds of the regularized flow (Corollary 5.8). In particular, an orbit that reaches the collision manifold from the (nonlinearly) stable manifold of $\mathscr{P}_{2}$, follows an heteroclinic orbit from $\mathscr{P}_{2}$ to $\mathscr{P}_{1}$, and then leaves the collision manifold along the (nonlinearly) unstable manifold of $\mathscr{P}_{1}$, experiences a phase shift of $\pi$ in the $\alpha$ coordinate, while maintaining a constant value for the $\psi$ coordinate. It is straightforward to verify that, in terms of the angular coordinates $(\phi, \theta)$ on the sphere, this corresponds to a collision-transmission trajectory, as defined in Definition 1.1

This result shows that, at least locally around the singularity, the dynamics is not affected by the curvature of the space. Indeed the same notion of regularization of the flow for a single logarithmic center on the plane has already been proved, among others, in [4] with a completely different technique.

Remember that we placed the vortex at the point $Q$ with coordinates $(\phi, \theta)=(\pi / 2, \pi / 2)$ and that in terms of the coordinates $(\phi, \theta)$ the dynamics on the sphere is governed by the Hamiltonian $H_{g e o}$, given in (12). Also recall the definition of vortex half-sphere, antivortex half-sphere, vortex-parallel, vortex-meridian already given in Sect. 1.

In the attractive case, the results of Lemma 5.1 can be rephrased as follows.

Theorem 6.1. If $h \geq h_{2}$ then the motion is allowed everywhere on the sphere. If $h<h_{2}$ the motion is allowed only in a sphere cap containing the vortex point whose boundary is a vortex-parallel that lies on the vortex half-sphere for $h<h_{1}$ or in the antivortex half-sphere if $h_{2}<h<h_{1}$. Moreover the zero velocity manifold consists in one of the vortex parallel when $h<h_{2}$, it reduces to the antipodal point when $h=h_{2}$ and it is empty for $h>h_{2}$.

In order to understand the global dynamics it is useful to show the existence of a second conserved quantity, analogous to the angular momentum for planar dynamics. To this aim it is convenient to move the vortex point at the north pole, i.e. $Q=(0,0,2 R)$ (or, equivalently, to redefine the parameterization of the sphere). Obviously this does not change the dynamics. Note that the curves $\{\theta=$ const $\}$ and $\{\phi=$ const $\}$ now respectively correspond to the vortex parallels and to the vortex meridians.

In this setting, the geodesic distance is simply $d_{\boldsymbol{S}}(P, Q)=R(\pi-\theta)$, yielding the Hamiltonian

$$
\mathcal{H}_{\text {geo }}\left(\theta, \phi, p_{\theta}, p_{\phi}\right)=\frac{1}{2 R^{2}}\left(\frac{1}{\sin ^{2} \theta} p_{\phi}^{2}+p_{\theta}^{2}\right)+\frac{\Gamma}{4 \pi} \log (R(\pi-\theta)) .
$$


and the dynamical system

$$
\left\{\begin{array}{l}
\dot{\phi}=\frac{1}{R^{2} \sin ^{2} \theta} p_{\phi} \\
\dot{\theta}=\frac{p_{\theta}}{R^{2}} \\
\dot{p}_{\phi}=0 \\
\dot{p}_{\theta}=\frac{\cos \theta}{R^{2} \sin ^{3} \theta} p_{\phi}^{2}+\frac{\Gamma}{4 \pi} \frac{1}{\pi-\theta}
\end{array} .\right.
$$

In the Euclidean case, we get

$$
\mathcal{H}_{\text {euc }}\left(\theta, \phi, p_{\theta}, p_{\phi}\right)=\frac{1}{2 R^{2}}\left(\frac{1}{\sin ^{2} \theta} p_{\phi}^{2}+p_{\theta}^{2}\right)+\frac{\Gamma}{8 \pi} \log \left(2 R^{2}(1+\cos \theta)\right) .
$$

Going to the Lagrangian formulation, we can write the Euler-Lagrange equations

$$
\begin{gathered}
\frac{d}{d t}\left(R^{2} \dot{\phi} \sin ^{2} \theta\right)=0 \\
R^{2} \ddot{\theta}-R^{2} \sin \theta \cos \theta \dot{\phi}^{2}+\frac{\Gamma}{4 \pi} \frac{1}{\pi-\theta}=0 .
\end{gathered}
$$

The first shows the existence of a conserved quantity, namely the spherical angular-momentum $l=R^{2} \sin ^{2} \theta \dot{\phi}$. It follows that

Lemma 6.2. A necessary condition for a solution to either collide with the vortex or to reach the antipodal point is $l=0$.

Proof. Writing the energy relation in terms of $(\phi, \theta, \dot{\phi}, \dot{\theta})$ and substituting $\dot{\phi}=$ $\frac{l}{R^{2} \sin ^{2} \theta}$, it follows that, for a given value of the spherical angular momentum $l$, a solution exists only for those $\theta$ satisfying

$$
2 R^{2} h \sin ^{2} \theta-\frac{\Gamma}{4 \pi} R^{2} \sin ^{2} \theta \log (R(\pi-\theta))-l^{2} \geq 0 .
$$

Since the vortex is placed at $\theta=\pi$ and the antipodal point is at $\theta=0$, it follows that if either a collision occurs, or the antipodal point is reached, then $l^{2} \leq 0$.

Looking at the system (30), one can easily prove the existence of particular solutions as depicted in Fig. 2.

Lemma 6.3. (i) Any vortex-parallel on the vortex half-sphere is the support of a periodic orbit.

(ii) The vortex-meridians are flow-invariant.

Proof. For any $\boldsymbol{\theta} \in\left(\frac{\pi}{2}, \pi\right)$ the curve

$$
\gamma_{\boldsymbol{\theta}}(t):=\left(\phi, \theta, p_{\phi}, p_{\theta}\right)(t)=\left(\phi_{0}+t \frac{\boldsymbol{p}_{\boldsymbol{\phi}}}{R^{2}(\sin \boldsymbol{\theta})^{2}}, \boldsymbol{\theta}, \boldsymbol{p}_{\boldsymbol{\phi}}, 0\right)
$$

with $\boldsymbol{p}_{\boldsymbol{\phi}}{ }^{2}=-\frac{\Gamma}{4 \pi} \frac{R^{2} \sin ^{3} \boldsymbol{\theta}}{(\pi-\boldsymbol{\theta}) \cos \boldsymbol{\theta}}$ is a solution of the system. Note that the previous relation can not be satisfied if $\boldsymbol{\theta} \in(0, \pi / 2]$, which implies that only the vortex-parallels placed in the vortex half-sphere are support of periodic orbits. Moreover the period of $\gamma_{\boldsymbol{\theta}}(t)$ tends to zero as $\boldsymbol{\theta}$ goes to $\pi / 2$ or $\pi$. This proves 
statement $(i)$. Statement $(i i)$ immediately follows by noting that any initial data $\left(\phi, \theta, p_{\phi}, p_{\theta}\right)(0)=\left(\phi^{0}, \theta^{0}, 0, p_{\theta}^{0}\right)$ leads to an orbit traveling on the $\left\{\phi=\phi_{0}\right\}$ vortex meridian.

The above discussion provides a complete picture of the global dynamics. Any solution with non-zero angular momentum will never end up into the collision neither it will reach the antipodal point. Otherwise, in case $l=0$, the orbit is moving on the vortex-meridians and it will fall into the vortex point. By extending the singular trajectories as in Definition 1, it follows that collision-transmission solutions behave in three different ways, depending on the energy level $h$ : if $h<h_{2}$, after the ejection from the singularity, the particle reaches the zero velocity manifold, then it reverses the motion, falls back into the vortex point and repeats the motion on the same vortex-meridian but in the opposite direction. If $h \geq h_{2}$ the trajectory will reach the antipodal point. In the case of the Euclidean logarithmic potential the dynamics is well defined even at the antipodal point, which is a rest point. Then we have that if $h=h_{2}$ the ejection orbit is an heteroclinic orbit between a point of $\mathscr{P}_{2}$ and the antipodal rest point (which is the only rest point on the zero-velocity manifold). If $h>h_{2}$, after the ejection, the orbits travels along a vortex meridian, passes through the antipodal rest point and continues the motion on the opposite meridian until it falls down again into the singularity. Then it repeats periodically the same orbit.

\section{Acknowledgements}

The authors thank the anonymous referee for her/his criticisms which deeply improved the paper, suggesting to consider the geodesic distance induced by the Riemannian metric in the definition of the potential function.

\section{Appendix A. Useful limits}

In this appendix we list some asymptotic limits of the functions appearing in the equation of motion in McGeheee coordinates. They are useful to compute the spectrum of the linearization at the fixed points.

\section{On the total collision manifold $r=0$}

All the limits below are computed with respect to $r$ and are uniform with respect to the other variables. For the function $\hat{E}$, we have:

$$
\lim _{r \rightarrow 0^{+}} \hat{E}(h, r, \alpha)=\frac{\Gamma R^{2}}{2 \pi} \quad \lim _{r \rightarrow 0^{+}} \partial_{r} \hat{E}(h, r, \alpha)=0 \quad \lim _{r \rightarrow 0^{+}} \partial_{\alpha}{ }^{\wedge} E(h, r, \alpha)=0
$$

For the functions $\boldsymbol{a}, \boldsymbol{b}$, we have

$$
\begin{gathered}
\lim _{r \rightarrow 0^{+}} \boldsymbol{a}(r, \alpha)=1, \quad \lim _{r \rightarrow 0^{+}} \partial_{r} \boldsymbol{a}(r, \alpha)=0, \quad \lim _{r \rightarrow 0^{+}} \partial_{\alpha} \boldsymbol{a}(r, \alpha)=0 . \\
\lim _{r \rightarrow 0^{+}} \boldsymbol{b}(r, \alpha)=0, \quad \lim _{r \rightarrow 0^{+}} \partial_{r} \boldsymbol{b}(r, \alpha)=0 .
\end{gathered}
$$


For the functions $\boldsymbol{a}_{x}, \boldsymbol{b}_{x}, \boldsymbol{a}_{y}, \boldsymbol{b}_{y}$, we have

$$
\begin{gathered}
\lim _{r \rightarrow 0^{+}} \boldsymbol{a}_{x}(r, \alpha)=0 \quad \lim _{r \rightarrow 0^{+}} \boldsymbol{a}_{y}(r, \alpha)=0 \quad \lim _{r \rightarrow 0^{+}} \partial_{r} \boldsymbol{a}_{x}(r, \alpha)=0 \\
\lim _{r \rightarrow 0^{+}} \boldsymbol{b}_{x}(r, \alpha)=0 \quad \lim _{r \rightarrow 0^{+}} \boldsymbol{b}_{y}(r, \alpha)=0 \quad \lim _{r \rightarrow 0^{+}} \partial_{r} \boldsymbol{b}_{x}(r, \alpha)=0 \\
\lim _{r \rightarrow 0^{+}} \frac{r^{3} e^{-1 / r^{2}} \boldsymbol{b}_{x}(r, \alpha)}{\boldsymbol{b}(r, \alpha)}=0 \\
\lim _{r \rightarrow 0^{+}} \partial_{\alpha} \boldsymbol{a}_{x}(r, \alpha)=0 \quad \lim _{r \rightarrow 0^{+}} \partial_{\alpha} \boldsymbol{a}_{y}(r, \alpha)=0 \\
\lim _{r \rightarrow 0^{+}} \partial_{\alpha} \boldsymbol{b}_{x}(r, \alpha)=0 \quad \lim _{r \rightarrow 0^{+}} \partial_{\alpha} \boldsymbol{b}_{y}(r, \alpha)=0 \\
\lim _{r \rightarrow 0^{+}} \frac{r^{3} e^{-1 / r^{2}} \boldsymbol{b}_{x}(r, \alpha) \partial_{\alpha} \boldsymbol{b}_{x}(r, \alpha)}{\boldsymbol{b}^{2}(r, \alpha)}=0, \\
\lim _{r \rightarrow 0^{+}} \frac{r^{3} e^{-1 / r^{2}} \boldsymbol{b}_{y}(r, \alpha) \partial_{\alpha} \boldsymbol{b}_{y}(r, \alpha)}{\boldsymbol{b}^{2}(r, \alpha)}=0 . \\
\lim _{r \rightarrow 0^{+}} \frac{r^{3} e^{-1 / r^{2}} \partial_{\alpha} \boldsymbol{b}_{y}(r, \alpha)}{\boldsymbol{b}^{2}(r, \alpha)}=0
\end{gathered}
$$

For the functions $z_{x}, z_{y}$, we have:

$$
\begin{gathered}
\lim _{r \rightarrow 0^{+}} z_{x}(r, \alpha, \psi)=\sqrt{\frac{\Gamma}{\pi}} 2 R^{2} \cos \psi, \quad \lim _{r \rightarrow 0^{+}} z_{y}(r, \alpha, \psi)=\sqrt{\frac{\Gamma}{\pi}} 2 R^{2} \sin \psi, \\
\lim _{r \rightarrow 0^{+}} \partial_{r} z_{x}(r, \alpha, \psi)=0, \quad \lim _{r \rightarrow 0^{+}} \partial_{\alpha} z_{x}(r, \alpha, \psi)=0,
\end{gathered}
$$

$\lim _{r \rightarrow 0^{+}} \partial_{\psi} z_{x}(r, \alpha, \psi)=-\sqrt{\frac{\Gamma}{\pi}} 2 R^{2} \sin \psi, \quad \lim _{r \rightarrow 0^{+}} \partial_{\psi} z_{y}(r, \alpha, \psi)=\sqrt{\frac{\Gamma}{\pi}} 2 R^{2} \cos \psi$,

$$
\lim _{r \rightarrow 0^{+}} \partial_{r} z_{y}(r, \alpha, \psi)=0, \quad \lim _{r \rightarrow 0^{+}} \partial_{\alpha} z_{y}(r, \alpha, \psi)=0 .
$$

As consequence of the above asymptotic behaviors it follows that

$$
\begin{gathered}
\lim _{r \rightarrow 0^{+}} A_{1}(r, \alpha, \psi)=0, \quad \lim _{r \rightarrow 0^{+}} A_{2}(r, \alpha, \psi)=0, \quad \lim _{r \rightarrow 0^{+}} B(r, \alpha, \psi)=0 \\
\lim _{r \rightarrow 0^{+}} \partial_{\alpha} A_{1}(r, \alpha, \psi)=0, \quad \lim _{r \rightarrow 0^{+}} \partial_{\alpha} A_{2}(r, \alpha, \psi)=0 \\
\frac{d r}{d \tau}=0, \quad \frac{d \alpha}{d \tau}=\sqrt{\frac{\Gamma}{2 \pi}} \frac{1}{R} \sin (\psi-\alpha) \\
\lim _{r \rightarrow 0} \frac{d}{d \tau} \sqrt{\hat{\hat{E}}}=0 .
\end{gathered}
$$


Denoting by $J:=\left(J_{i j}\right)_{i, j}$ the variational matrix on the total collision manifold it follows that

$$
\begin{aligned}
& J_{11}=0, \quad J_{12}=0, \quad J_{13}=0, \quad J_{32}=0, \quad J_{33}=0 \\
& J_{21}=0, \quad J_{22}=-\frac{\Gamma}{2 \pi} \cos (\psi-\alpha), \quad J_{23}=-J_{22}, \\
& \lim _{r \rightarrow 0^{+}} \partial_{\alpha \tau}^{2} \boldsymbol{a}(r, \alpha)=0, \quad \lim _{r \rightarrow 0^{+}} \partial_{\alpha \tau}^{2} \hat{E}(h, r, \alpha)=0 .
\end{aligned}
$$

The limit involved in the computation of the term $J_{31}$, in general may not exist. However at the restpoints $\psi=\alpha$ or $\psi=\alpha+\pi$ this limit actually exists and this implies that $J_{31}=0$.

\section{References}

[1] Ralph, A., Marsden, J.E.: Foundations of Mechanics, 2nd edn. Benjamin/Cummings, Ink. Massachusetts (1978)

[2] Vivina, B., Ferrario, D.L., Susanna, T.: On the singularities of generalized solutions to $n$-body-type problems. Int. Math. Res. Not. IMRN (2008)

[3] Castelli, R.: On the variational approach to the one and N-centre problem with weak forces. Ph.D. dissertation, University of Milano-Bicocca (2009)

[4] Roberto, C., Susanna, T.: On the regularization of the collision solutions of the one-center problem with weak forces. Discrete Contin. Dyn. Syst 31(4), 1197$1218(2011)$

[5] Diacu, F., Perez-Chavela, E., Reyes Victoria, J.G.: An intrinsic approach in the curved n-body problem: the negative curvature case. J. Differ. Equ. 252, 45294562 (2012)

[6] Florin, D.: Polygonal homographic orbits of the curved $n$-body problem. Trans. Am. Math. Soc. 364(5), 2783-2802 (2012)

[7] Florin, D., Perez-Chavela, E.: Homographic solutions of the curved 3-body problem. J. Differ. Equ. 250, 1747-1766 (2011)

[8] Florin, D.: On the singularities of the curved $n$-body problem. Trans. Am. Math. Soc. 363(4), 2249-2264 (2011). http://arxiv.org/abs/0812.3333

[9] Florin, D., Perez-Chavela, E., Santoprete, M.: The $n$-body problem in spaces of constant curvature. http://arxiv.org/abs/0807.1747

[10] Easton, R.: Regularization of vector fields by surgery. J. Differ. Equ. 10, 92-99 (1971)

[11] Ferrario, D.L., Portaluri, A.: On the dihedral $n$-body problem. Nonlinearity 21(6), 1307-1321 (2008)

[12] Bellettini, G., Fusco, G., Gronchi, G.F.: Regularization of the two-body problem via smoothing the potential. Commun. Pure Appl. Anal. 2(3), 323-353 (2003) 
[13] Hooverman, R.H.: Charged particle orbits in a logarithmic potential. J. Appl. Phys 34, 3505-3508 (1963)

[14] Kenig, C.E., Ponce, G., Vega, L.: On the interaction of nearly parallel vortex filaments. Commun. Math. Phys. 243, 471-483 (2003)

[15] Levi-Civita, T.: Sur la régularisation du problème des trois corps. Acta Math. 42(1), 99-144 (1920)

[16] McGehee, R.: Triple collision in the collinear three-body problem. Invent. Math. 27, 191-227 (1974)

[17] Newton, P.: The $N$-vortex problem. Analytical techniques. Applied Mathematical Sciences, vol. 145. Springer, New York (2001)

[18] Stoica, C., Font, A.: Global dynamics in the singular logarithmic potential. J. Phys. A 36, 7693-7714 (2003)

[19] Touma, J., Tremaine, S.: A map for eccentric orbits in non-axisymmetric potentials. MNRAS 292, 905-932 (1997)

[20] Vidal, C.: The tetrahedral 4-body problem with rotation. Celestial Mech. Dyn. Astron. 71(1):15-33 (1999)

Roberto Castelli

Basque Center for Applied Mathematics (BCAM)

Alameda de Mazarredo, 14

48009 Bilbao

Spain

e-mail: rcastelli@bcamath.org

Francesco Paparella and Alessandro Portaluri

Dipartimento di Matematica e Fisica "Ennio De Giorgi"

Ex-collegio Fiorini

University of Salento

73100 Lecce

Italy

e-mail: alessandro.portaluri@unisaleno.it

Francesco Paparella

e-mail: francesco.paparella@unisalento.it

Received: 11 February 2012.

Accepted: 21 June 2012. 